\title{
Actinomycosis of the Appendix Mimicking Cecal Tumor Treated by Single-Port Laparoscopic Approach
}

\author{
In Soo Cho ${ }^{1}$, Sung Uk Bae ${ }^{1}$, Hye Ra Jung ${ }^{2}$, Kyung Sik Park ${ }^{3}$, Woon Kyung Jeong ${ }^{1}$, Seong Kyu Baek ${ }^{1}$ \\ ${ }^{1}$ Department of Surgery, Keimyung University Dongsan Medical Center, Keimyung University School of Medicine, Daegu; ${ }^{2}$ Department of \\ Pathology, Keimyung University Dongsan Medical Center, Keimyung University School of Medicine, Daegu; ${ }^{3}$ Division of Gastroenterology \\ and Hepatology, Department of Internal Medicine, Keimyung University Dongsan Medical Center, Keimyung University School of Medicine, \\ Daegu, Korea
}

Actinomycosis is an inflammatory disease with various clinical presentations including inflammation and formation of masses. There are several reports suggesting the infiltrative mass-like nature of actinomycosis that is misunderstood as a tumor. A 39-year-old male clinically presented with a fungating mass-like lesion during colonoscopy for healthcare screening. Biopsy was performed for the lesion, and chronic inflammation was diagnosed. Abdominal computed tomography (CT) suggested severe edematous changes in the appendix with an appendicolith, suspected chronic inflammation, and wall thickening of the cecal base, but malignancy could not be definitively ruled out. The patient underwent a laparoscopic single-port cecectomy based on the possibility of cecal cancer. The final biopsy was diagnosed as actinomycosis, and the patient was prescribed antibiotics and showed no recurrence in the follow-up CT scan. We present this rare case of mass-like appendiceal actinomycosis treated with the single-port laparoscopic method.

Keywords: Appendiceal actinomycosis; Single-port appendectomy; Cecal mass

\section{INTRODUCTION}

Actinomycosis is a chronic, suppurative, granulomatous disease caused by an anaerobic gram-positive bacterium, Actinomyces israelii, manifesting as fistulae, sinuses, inflammatory pseudotumors, and abscess [1]. The estimated population prevalence of actinomycosis is one case per 40-119,000, with a recent decrease in prevalence. The majority of clinical disease (55\%) is cervicofacial, with only $20 \%$ occurring in the abdominopelvic region and $15 \%$ characterized in the pulmonothoracic region [2].

There are various case reports suggesting unusual pathologic presentation of actinomycosis [3-5]. Some reports have described

Received: May 5, 2019 • Revised: Aug 1, 2019 - Accepted: Aug 10, 2019 Correspondence to: Sung Uk Bae, M.D.

Department of Surgery, Keimyung University School of Medicine, Dongsan Medical Center, 1035 Dalgubeol-daero, Dalseo-gu, Daegu 42601, Korea

Tel: +82-53-250-7114, Fax: +82-53-250-7114

E-mail: sabiston0000@hanmail.net

ORCID: https://orcid.org/0000-0002-7876-4196

(c) 2021 The Korean Society of Coloproctology

This is an open-access article distributed under the terms of the Creative Commons Attribution NonCommercial License (https://creativecommons.org/licenses/by-nc/4.0) which permits unrestricted noncommercial use, distribution, and reproduction in any medium, provided the original work is properly cited. the radiological findings of abdominopelvic actinomycosis as an infiltrative mass with unusual aggressiveness. Some reports of appendiceal actinomycosis have described wall thickening and periappendiceal inflammation with contrast enhancement, which could be misdiagnosed as colon malignancy. Surgical resection is necessary in some cases as an adjunct to medication and has been performed in an open manner, though this has recently changed to a laparoscopic approach [6].

Since laparoscopic appendectomy was first described in 1983, this approach was rapidly accepted as an adequate option for various kinds of diseases [7]. As minimally invasive surgery has developed, minimizing surgical trauma and improving cosmetic outcomes have evolved as topics of active discussion and have led to development of single-port laparoscopic surgery (SPLS) for treatment of a variety of conditions including acute appendicitis [8-11].

Recently, we encountered a patient who was referred from a local medical center with a cecal mass whose final biopsy revealed actinomycosis after cecectomy. We report a rare case of actinomycosis of the appendix mimicking a cecal tumor treated through a single-port laparoscopic approach. 


\section{CASE REPORT}

A 39-year-old man with no previous illness visited a local medical center for healthcare checkup. He exhibited no other symptoms and was observed to harbor a mass-like lesion on which a punch biopsy was performed. The initial biopsy revealed an inflammatory lesion; to further evaluate for the possibility of cancer, he was referred to a tertiary referral center. Informed consent was obtained from the patient, and the case was approved by the Institutional Review Board (No. 2019-02-046).

The patient underwent repeat colonoscopy, biopsy, and abdominal computed tomography (CT) in the gastroenterology department to rule out malignancy. The colonoscopy, which was performed for confirmation, revealed a fungating mass-like lesion with hyperemic mucosa around the appendiceal orifice that was not compatible with the usual findings of colonic adenocarcinoma (Fig. 1B). The abdominal CT findings were described as severe edematous changes in the appendix, with appendicolith, suspected chronic inflammation, and wall thickening of the cecal base (Fig. 1A). A radiologist recommended surgical evaluation to exclude cecal malignancy. Pathology of the colonoscopic biopsy revealed simple inflammatory change with chronic colitis. Regarding the differential diagnosis for Crohn's disease, there were no specific colonoscopic findings such as skip lesions, cobblestoning, ulcerations, or strictures and no pathologic findings including transmural inflammation, granulomas, or crypt abscesses to justify that diagnosis. Even though the colonoscopic and radiologic findings did not direct the diagnosis to colon adenocarcinoma, a gastroenterologist recommended surgical evaluation of the mass to rule out malignancy of the colon. After the preoperative workup and colorectal multidisciplinary team meeting, we planned to perform single-port laparoscopic cecectomy followed by right hemicolectomy if the final surgical biopsy report revealed signs of cancer.

Regarding surgical technique, a single vertical incision was made through the umbilical skin, and an S Port (Eraesi Medical, Hanam, Korea) was placed in the abdominal cavity through the umbilical incision. The mesoappendix was divided through sequential use of a Covidien Sonicision (Covidien, Mansfield, MA, USA) device. The cecum was divided with application of an Endo-GIA Stapler (Covidien). The appendix was removed through the umbilical incision and placed into the sterile bag component of the single-port (Fig. 2).

The resected appendix was transported to the pathology department. Microscopic findings revealed a thickened wall and purple sulfur granules in the submucosa and muscular wall as well as in the lumen (Fig. 3). As a result, this patient was diagnosed with appendiceal actinomycosis. After diagnosis, the patient was referred to the medical department for further antibiotic treatment for actinomycosis. After undergoing 1 month of treatment with Augmentin (Amoxicillin-Clavulanate), the patient's follow-up CT exhibited no signs of recurring infection.

\section{DISCUSSION}

The most common pathogen of pathogenic actinomycosis in humans is $A$. israelii. These organisms are indigenous to the oral cavity, gastrointestinal tract, and genital tract, with opportunistic infections occurring when the mucosal barrier is broken, leading to multiple abscesses, fistulae, and mass lesions. Human actinomycosis commonly occurs in three distinct forms, which may occasionally overlap. The majority of clinical disease (55\%) is cervicofacial, with only $20 \%$ occurring in the abdominopelvic regions mentioned previously [2]. The ileocecal area is the abdominal site
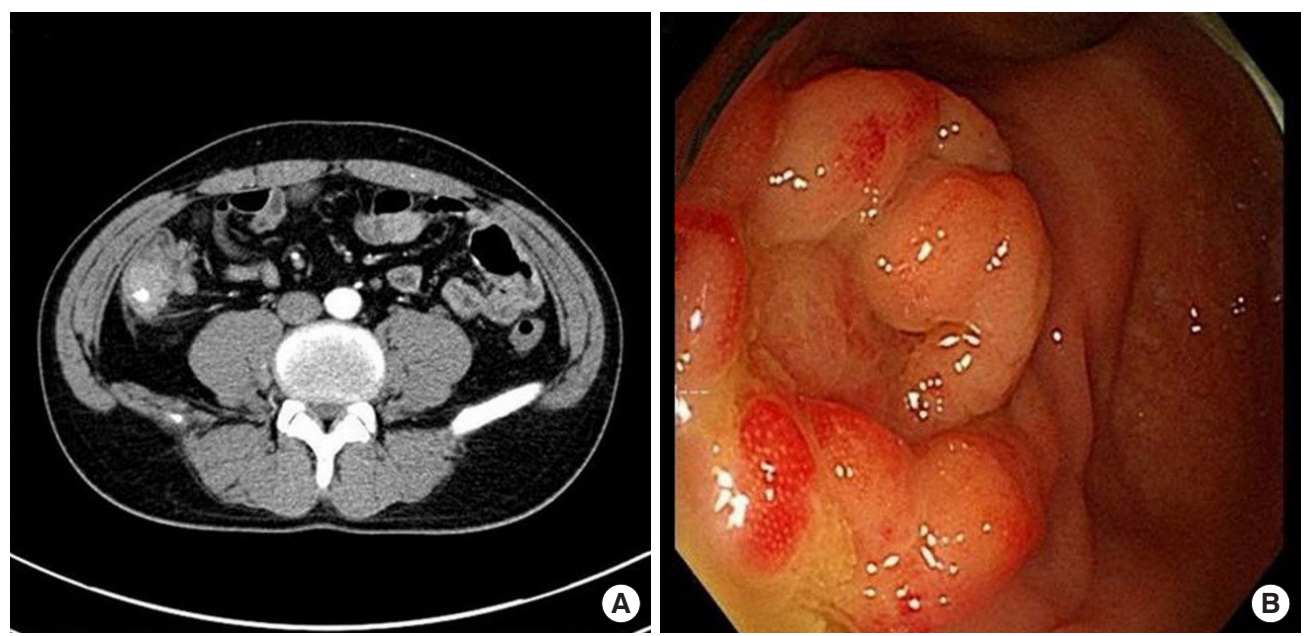

Fig. 1. Preoperative evaluation. (A) Axial view of the abdominal computed tomography. Severe edematous changes in the appendix with appendicolith, suspected chronic inflammation, and wall thickening of the cecal base that was suspicious for cecal tumor. (B) Colonoscopic finding. Mass-like lesion around the appendiceal orifice. 

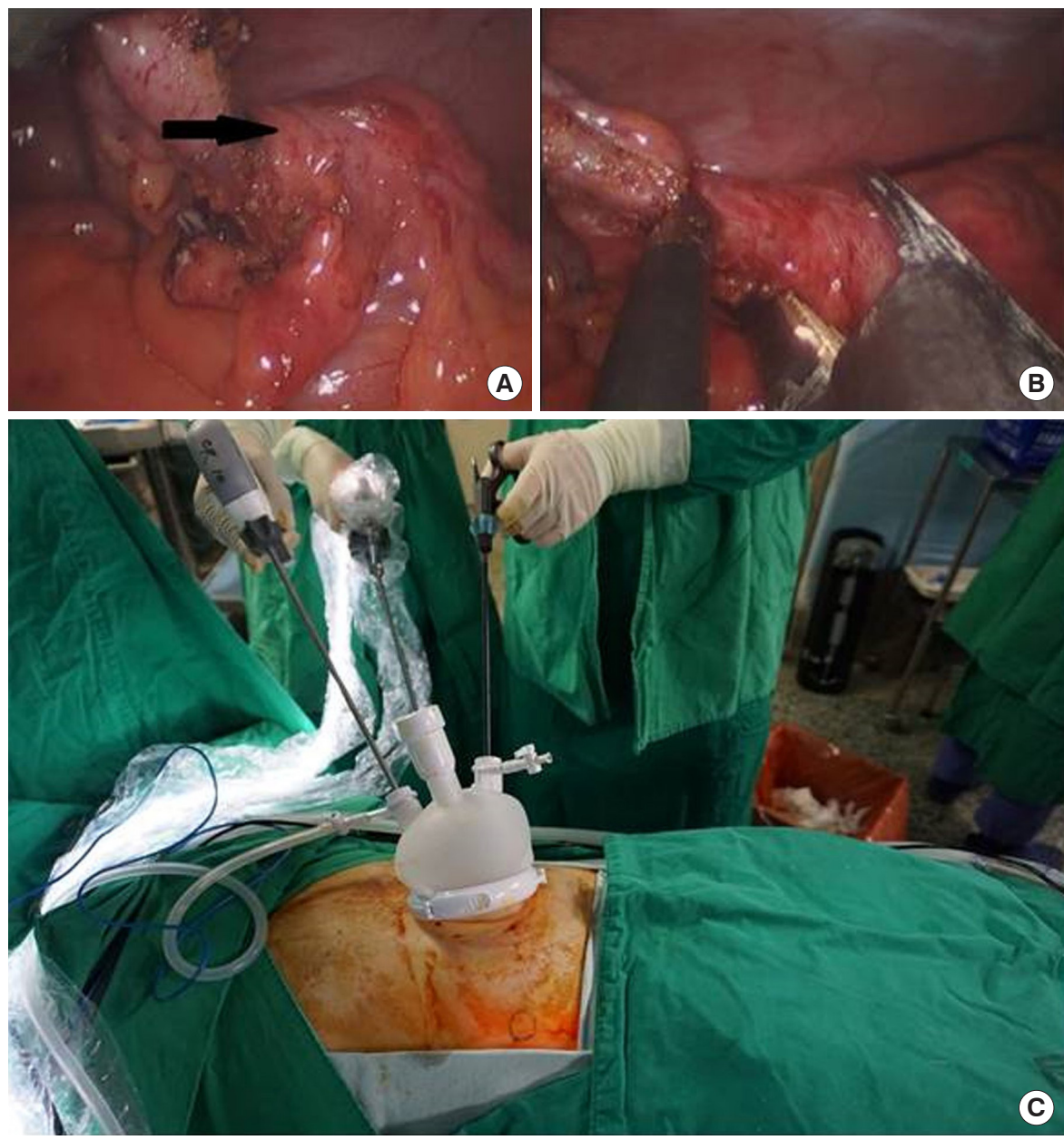

Fig. 2. Intraoperative findings. (A) Swollen appendicealbase (arrow). (B) Cecectomy with a laparoscopic linear stapler. (C) Exterior view of the inserted single-port and operator.
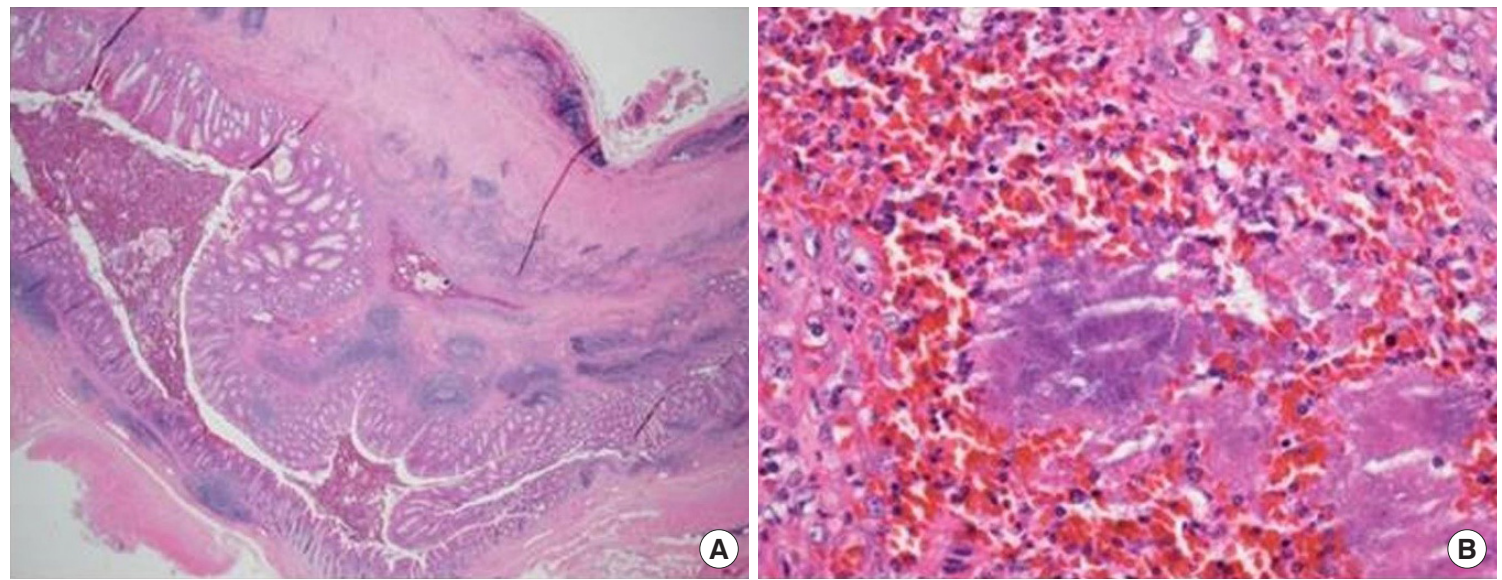

Fig. 3. Surgical pathology. (A) A thick appendiceal wall is noted. In the submucosa, eosinophilic fibrotic deposits and irregular spaces filled with extravasated red blood cells (RBCs) and inflammatory cells were present $(\mathrm{H} \& \mathrm{E}, \times 12.5)$. (B) In the space, extravasated RBCs and variable inflammatory cells including lymphocytes and neutrophils were present. Typical actinomycetes colonies (sulfur granules) composed of basophilic radiating filaments were also observed $(\mathrm{H} \& \mathrm{E}, \times 400)$. 
most commonly affected, with predomination of the appendix. Deshmukh and Heaney [12] reviewed 205 cases of abdominal actinomycosis, and two-thirds involved the appendix and cecum. Most ileocecal type actinomycosis presents with variable symptoms. Two of the most common symptoms are abdominal tenderness due to inflammation and a palpable mass. Differential diagnosis of this disease includes acute appendicitis or Crohn disease as an acute presentation of inflammatory bowel disease and carcinoma of the cecum or ascending colon if a mass is the predominant feature.

With the patient presenting no symptoms but possessing a masslike lesion in the colonoscopic findings, benign neoplastic surgery was performed. For minimal invasiveness, we performed singleport laparoscopic partial cecectomy using a laparoscopic linear stapler to achieve negative safe margins. There was no postoperative ileocecal narrowing, and safe margins were achieved. Benign appendiceal neoplasms are treated with several surgical techniques including SPLS. Unlike single-port appendectomy where the application and feasibility have been studied extensively, not many single-port cecectomy studies have been conducted. Park et al. [13] reported a case series of successful outcomes with 16 cases of appendiceal mucoceles treated with SPLS. With greater success in cosmetic outcomes, less postoperative pain, and increased procedural safety, we believe that SPLS cecectomy is a feasible treatment option for benign appendiceal and cecal masses.

When typical yellow sulfur granules are grossly observed during surgery, actinomycosis can be strongly presumed. Microscopically, basophilic bacterial colonies with radiating features (typical microscopic findings of sulfur granules) are pathognomonic features of actinomycosis. In this case, we identified many basophilic sulfur granules surrounded by many kinds of inflammatory cells in the appendiceal wall, indicating actinomycosis.

Regarding medical treatment, all actinomyces species remain sensitive to most common antibiotics. Therefore, the treatment of choice remains high-dose antibiotic therapy for prolonged periods, as the intense desmoplastic reaction is associated with actinomycosis-limiting drug penetration [14]. Penicillin is considered the antibiotic of choice, since there are not many forms of actinomycosis that exhibit antibiotic resistance [15]. Surgical resection may not be needed and may not be ideal for intraperitoneal diseases, where it may be difficult to confidently resect all infected tissue. It is also difficult to distinguish adenocarcinoma from infectious bowel disease with mass forming lesion such as abdominal actinomycosis in the surgical field, which confers the risk of retained cancer tissue when left untreated. Minimal invasive surgery needs to be performed for both diagnosis and treatment for this kind of disease and we think single-port surgery is feasible in this manner.

\section{ACKNOWLEDGMENTS}

This work was supported by a National Research Foundation of Korea (NRF) grant funded by the Korean government (MSIP) (No. 2017R1C1B5076880).

\section{REFERENCES}

1. Koren R, Dekel Y, Ramadan E, Veltman V, Dreznik Z. Periappendiceal actinomycosis mimicking malignancy report of a case. Pathol Res Pract 2002;198:441-3.

2. Bennhoff DF. Actinomycosis: diagnostic and therapeutic considerations and a review of 32 cases. Laryngoscope 1984;94:1198217.

3. Oh HB, Abdul Malik MH, Keh CH. Pilonidal abscess associated with primary actinomycosis. Ann Coloproctol 2015;31:243-5.

4. Jee SB, Jang KS, Kim SH, Kim CJ. Actinomycosis of the appendix: a case report. J Korean Soc Coloproctol 2010;26:233-7.

5. Lee RA, Han SH, Kim, Kim OY. The colonic obstruction due to actinomycosis. J Korean Soc Coloproctol 1998;14:649-54.

6. Lee SY, Kwon HJ, Cho JH, Oh JY, Nam KJ, Lee JH, et al. Actinomycosis of the appendix mimicking appendiceal tumor: a case report. World J Gastroenterol 2010;16:395-7.

7. Semm K. Endoscopic appendectomy. Endoscopy 1983;15:59-64.

8. Bae SU, Jeong WK, Baek SK. Single-port laparoscopic complete mesocolic excision and central vascular ligation for caecal cancer with apical lymph node metastasis: a video vignette. Colorectal Dis 2018;20:643-4.

9. Bae SU, Jeong WK, Baek SK. Single-port laparoscopic interval appendectomy for perforated appendicitis with a periappendiceal abscess. Ann Coloproctol 2016;32:105-10.

10. Bae SU, Min BS, Baik SH, Kim NK, Hur H. Single-port laparoscopic total extraperitoneal inguinal hernia repair without fixation of the mesh. J Minim Invasive Surg 2016;19:25-31.

11. Bae SU, Jeong WK, Baek SK. Current status of robotic single-port colonic surgery. Int J Med Robot 2017;13.

12. Deshmukh N, Heaney SJ. Actinomycosis at multiple colonic sites. Am J Gastroenterol 1986;81:1212-4.

13. Park KB, Park JS, Choi GS, Kim HJ, Park SY, Ryuk JP, et al. Singleincision laparoscopic surgery for appendiceal mucoceles: safety and feasibility in a series of 16 consecutive cases. J Korean Soc Coloproctol 2011;27:287-92.

14. Garner JP, Macdonald M, Kumar PK. Abdominal actinomycosis. Int J Surg 2007;5:441-8.

15. Turck M, Smith J. Prospective pricing system and its effect on the principles and practice of infectious diseases. Am J Med 1985;78: 23-8.

\section{CONFLICT OF INTEREST}

No potential conflict of interest relevant to this article was reported. 